\title{
Angioplastía primaria en infarto agudo al miocardio en arteria coronaria derecha con origen anómalo. Caso clínico
}

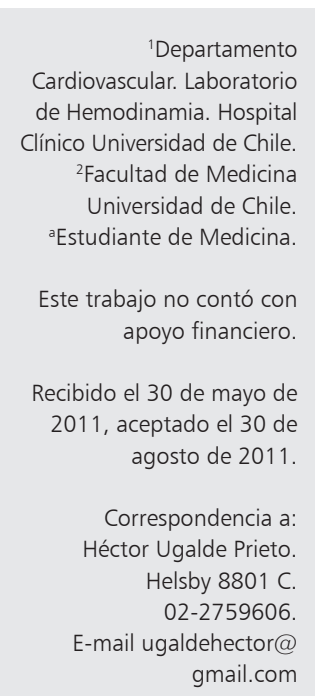

\author{
HÉCTOR UGALDE ${ }^{1}$, MACARENA MUÑOZ2,a, \\ DIEGO UGALDE ${ }^{2, a}$, SEBASTIÁN GARCÍA ${ }^{1}$
}

\section{Primary angioplasty for acute myocardial infarction with an anomalous origin of right coronary artery. Report of one case}

Anomalous origin of coronary arteries is a rare anatomical defect and its association with acute myocardial infarction is unusual. We report a 58-year-old male with ST-Segment elevation. Myocardial infarction of the inferior wall caused by a total occlusion on the proximal third of an anomalous right coronary artery, that was effectively treated with primary angioplasty with stent placement. The patient had a favorable outcome and is asymptomatic after five years of follow up.

(Rev Med Chile 2012; 140: 88-92).

Key words: Angioplasty; Coronary angiography; Coronary stenosis.
E 1 origen anómalo de las arterias coronarias es un tipo de defecto poco usual, de carácter congénito ${ }^{1}$, que puede presentarse de manera aislada o asociada a otras malformaciones cardiacas $^{1,2}$. Se han descrito diferentes prevalencias en registros de necropsias, series coronariográficas y tomográficas en población seleccionada ${ }^{3-5}$. Clínicamente se asocia a muerte súbita (MS) en esfuerzo siendo su principal manifestación clínica, destacando su presentación en pacientes jóvenes $y$ atletas ${ }^{6,7}$. Además pueden presentarse síntomas isquémicos e infarto agudo al miocardio (IAM) de manera anecdótica, existen pocos casos descritos de IAM en arteria coronaria anómala (ACA) que hayan sido tratados con angioplastía primaria (APP), y en nuestra literatura nacional no se conoce, lo que motiva este reporte. Se describe el caso de un paciente con infarto agudo al miocardio en arteria coronaria con origen anómalo sometido a angioplastía primaria.

\section{Descripción del caso}

Paciente de sexo masculino de 58 años de edad sin antecedentes mórbidos, que en agosto del año
2001, inició cuadro de dolor precordial progresivo, de carácter opresivo, de intensidad inicial en escala de evaluación análoga (EVA) 5 de 10, irradiado a brazo izquierdo, acompañado de sudoración profusa y vómitos. Consultó a las 3 horas de evolución de sus síntomas. A su ingreso se constató vigil y orientado, con frecuencia cardiaca 62 latidos por minuto, presión arterial $149 / 89 \mathrm{mmHg}$, frecuencia respiratoria 16 por minuto con dolor precordial en intensidad EVA 9/10. Se realiza electrocardiograma (ECG) de 12 derivaciones (Figura 1) que mostraba supradesnivel del segmento $S T$ en pared inferior y V1. Se diagnosticó IAM con supradesnivel del segmento ST, se inició manejo médico y se activó laboratorio de hemodinamia. Se realizó coronariografía (Figura 2) a los 45 minutos de ingreso con ausencia de visualización de arteria coronaria derecha (ACD) (2A), arterias descendente anterior (ADA) y circunfleja (ACx) sin lesiones. Se sospechó oclusión ostial de ACD, pero una segunda opinión planteó la existencia de un posible nacimiento anómalo, por lo que se decidió realizar aortografía que mostró la $\mathrm{ACD}$ con nacimiento anómalo en seno coronario izquierdo alto con lesión $100 \%$ en su tercio medio lo que se 
Angioplastía primaria en IAM en coronaria de origen anómalo - H. Ugalde et al

confirmó con inyección selectiva realizada con cateter amplaz (2B). Se realizóAPP con implante de stent (2C), lográndose flujo TIMI 3 en ACD, sin lesión residual(2D). ECG post-reperfusión mostró normalización del segmento ST. La curva enzimática se muestra en la Tabla 1.

Evolucionó hemodinámicamente estable, sin presentar complicaciones en la evolución de su IAM, trasladándose a las 72 horas a sala. Ecocardiografía mostró aquinesia del tercio basal de la pared inferior y septum posterior, con hipoquinesia moderada del tercio medio y apical posterior, con una función sistólica conservada en rango normal, leve dilatación de la aurícula derecha con ventrículo derecho de tamaño y motilidad normal. Evolucionó favorablemente y fue dado de alta al sexto día de hospitalización, recibiendo ácido acetil-salicílico, clopidogrel, atenolol, enalapril y simvastatina. Evolucionó asintomático y sin nuevos eventos a los 5 años de seguimiento.
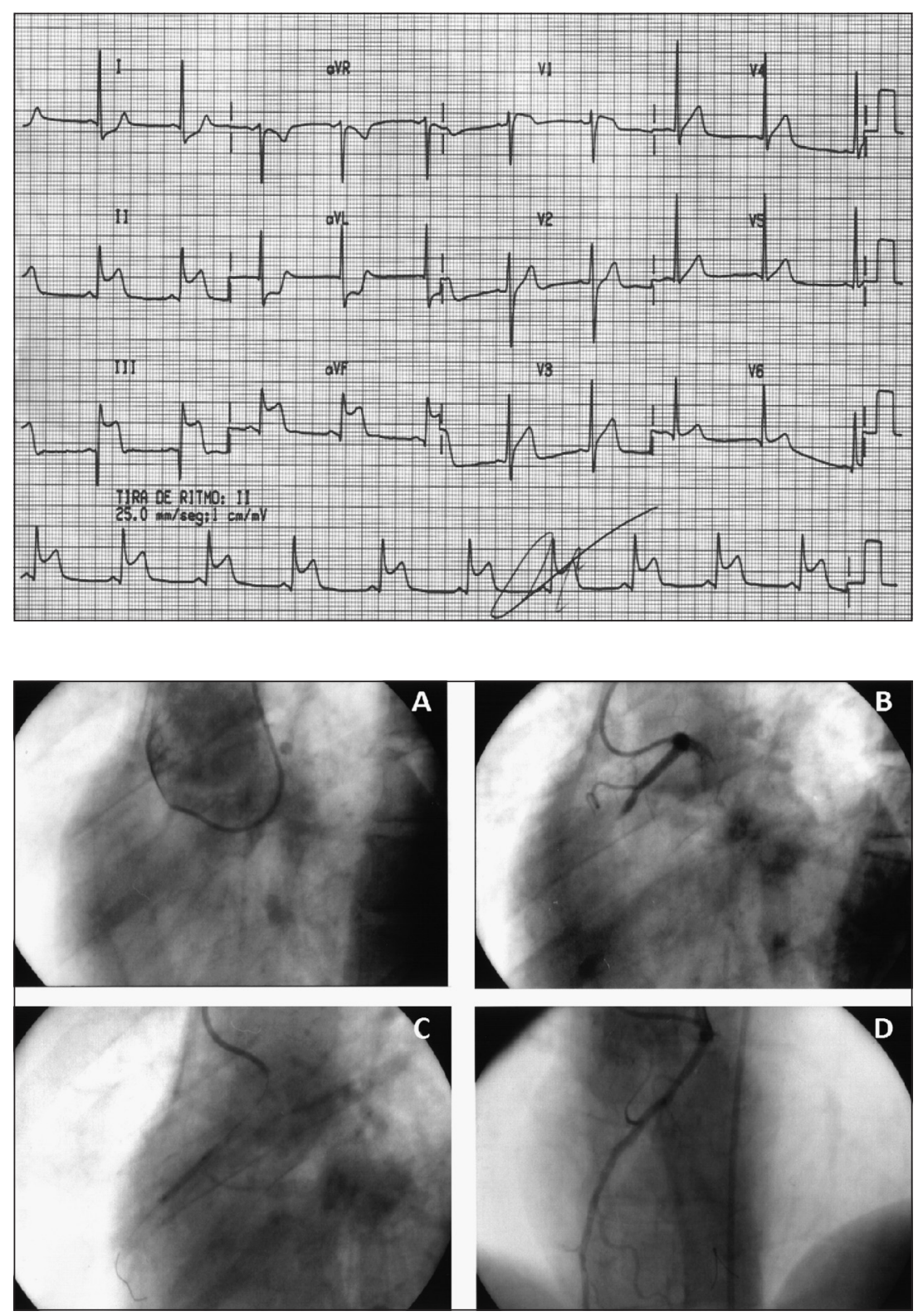

Figura 1. Electrocardiograma de 12 derivaciones que muestra supradesnivel del segmento ST en pared inferior(D2,D3,AVF).

Figura 2. Coronariografía y angioplastía primaria. 2A. Coronariografía que muestra ausencia de CD en el seno coronario derecho (Proyección oblicua anterior izquierda (P OAI) $45^{\circ}$ ). 2B. ACD con origen en seno coronario izquierdo alto con lesión 100\% en tercio medio (P OAl $45^{\circ}$ ). $\mathbf{2 C}$. Angioplastía Primaria en misma ACD con origen anómala (P OAI $45^{\circ}$ ). 2D. ACD con angioplastia e implante de stent en su tercio medio, sin lesión residual, flujo Timi 3 (P anteroposterior). 
Angioplastía primaria en IAM en coronaria de origen anómalo - H. Ugalde et al

Tabla 1. Curva enzimática: Se aprecia alza precoz, sugerente de reperfusión exitosa

\begin{tabular}{|lccccc|}
\hline & $\mathbf{4}$ horas & $\mathbf{1 2}$ horas & $\mathbf{2 4}$ horas & $\mathbf{4 8}$ horas & Valor normal laboratorio \\
\hline CK total $(\mathrm{U} / \mathrm{L})$ & 80 & 730 & 709 & 395 & $\leq 170$ \\
$\mathrm{CK}-\mathrm{MB}(\mathrm{U} / \mathrm{L})$ & 15 & 106 & 89 & 27 & $\leq 16$ \\
\hline
\end{tabular}

Nota: Horas desde inicio de dolor.

\section{Discusión}

El origen anómalo de las arterias coronarias corresponde a una variante en el nacimiento de los troncos coronarios desde un lugar anatómico poco usual (considerando como anomalía aquellas variantes que se observan en menos del 1\% de una población no seleccionada), que puede encontrarse asociada a malformaciones congénitas cardiacas o aisladamente ${ }^{1,2,8}$. Su incidencia se ha estudiado en distintos tipos de investigaciones, desde estudios de anatomía patológica, tomografía computarizada hasta grandes series coronariográficas. Se ha visto una incidencia de hasta 2,2\% de ACA en necropsias de series de malformaciones cardiacas ${ }^{8}$; mientras que en series coronariográficas se ha observado una incidencia que va desde 0,3 hasta $1,3 \%$, con casuísticas que analizan desde 3.700 hasta poco más de 126.000 procedimientos $^{3-5}$. En nuestro país existe un reporte reciente que observó una incidencia de $1,29 \%$ en una serie de 10.000 coronariografías ${ }^{9}$.

En términos anatómicos y angiográficos, una ACA puede describirse según el ostium que da su nacimiento (número y origen), su tronco principal (trayecto entre grandes vasos, tamaño), divisiones principales, extramurales e intramurales ${ }^{1,8}$. Se ha descrito además que la dominancia podría ser un factor pronóstico para establecer una anomalía como patológica o significativa en análisis postmortem $^{2}$. El caso presentado muestra un origen anómalo de la ACD con tronco de nacimiento en seno coronario contralateral y de implantación alta respecto a su seno coronario esperado.

Se considera la MS como la principal manifestación clínica, característicamente en jóvenes y deportistas, donde la primera causa de MS es la miocardiopatía hipertrófica con 36\%, seguida de las ACA hasta en $17 \% \%^{6,7}$. En casuísticas de necropsias con malformaciones cardiacas se describe la MS hasta en $60 \%$, desencadenada por esfuerzo en la mitad de los casos (en su mayoría atletas) con sintomatología previa como síncope, palpitaciones y dolor torácico ${ }^{8}$.
La asociación directa de ACA con enfermedad coronaria se ha estudiado en varias series, con resultados controversiales ${ }^{4,10}$. Se ha descrito en estudios de caso control que no existiría mayor asociación a enfermedad coronaria en comparación con la población sin $\mathrm{ACA}^{3,4}$, hecho que también se observa en la población chilena'. Se desconoce si existe mayor riesgo de IAM.

En cuanto a sexo, en la mayoría de las series descriptivas muestran que del total de ACA existe mayor proporción de sexo masculino ${ }^{3-5,10,11}$ (desde 70,6 hasta $87,2 \%$ del total de ACA), pudiendo existir un sesgo dada la mayor incidencia de patología coronaria en el hombre, siendo sometidos a más estudios angiográficos; además de existir poca precisión en la metodología de los estudios respecto a la población del mismo sexo sin ACA. No se ha observado correlación con antecedentes familiares ${ }^{4}$.

Su diagnóstico se realiza a través de angiografía coronaria, tomografía computada (TC), resonancia magnética $(\mathrm{RM})$ y análisis anatomopatológi$\mathrm{Co}^{3-5,12}$. El primero puede demostrar la presencia de un ostium, tronco coronario o la arteria coronaria en una ubicación anómala además de identificar enfermedad coronaria ateroesclerótica asociada y ofrecer alternativas terapéuticas dependiendo de la situación clínica y el motivo de estudio. Se ha descrito que la aortografía, puede facilitar su demostración angiográfica cuando un ostium coronario no puede ser cateterizable dada su "ausencia" en un primer intento ${ }^{13}$. La TC y la RM son muy útiles para el estudio tridimensional de las ACA y su relación con los grades vasos ${ }^{5,12,14,15}$.

El IAM en ACA se considera un hecho anecdótico. En pacientes sometidos a estudio coronariográfico $^{3,4}$ con hallazgo de ACA, se ha encontrado que hasta $22 \%$ de los pacientes tenía signos de infarto antiguo en ECG de 12 derivaciones, sin establecer una relación clara con la ACA existente. En pacientes con anomalía coronaria sintomática sometidos a resolución quirúrgica, 15\% presentaba historia de infarto antiguo atribuible a la anomalía ${ }^{16}$. 
La literatura de IAM tratado con APP en ACA es escasa, el primer caso fue descrito recién en el año $2004{ }^{17}$ y luego de este sólo se han descrito unos pocos más ${ }^{18,19}$, siendo este caso el primero descrito en el medio local y latinoamericano, también aparecen en la literatura algunos casos descritos de IAM en ACA que han sido manejados con cirugía ${ }^{20}$ o angioplastía diferida ${ }^{13}$. La descripción de este caso nos muestra la dificultad a la cual se puede enfrentar el cardiólogo intervencional en algunos de sus pacientes, aquí se relata el como el primer cardiólogo que enfrento el caso tuvo una forma de interpretación y como la ayuda del segundo medico permitió tomar una decisión diferente, medico que se había enfrentado recientemente a un caso similar en una situación no de urgencia, decisión que al final resulto la correcta. Además debe destacarse la dificultad técnica a la que se es sometido al enfrentar una intervención en un paciente con coronarias anómalas, y algunas formas de superar estas dificultades, como la utilización de catéteres especiales, de diferente forma, etc., como bien ha sido planteado en la literatura ${ }^{18}$, todos hechos que conocidos, permitirán superar los problemas que se presenten, como así ocurrió en este caso en particular.

\section{Conclusiones}

El caso presentado describe por primera vez en nuestro medio la terapia con angioplastía primaria exitosa en infarto agudo al miocardio debido a oclusión de una arteria coronaria derecha con origen anómalo en seno coronario izquierdo. Los síndromes coronarios agudos en arterias coronarias anómalas son un hecho excepcional que debe tenerse en cuenta durante procedimientos de cardiología invasiva donde el angiografista puede no encontrar la anatomía esperada, donde la aortografía es útil en el diagnóstico y existen diferentes técnicas recomendadas para enfrentar estos casos de sospecha de arterias coronarias anómalas para lograr una terapéutica exitosa.

\section{Referencias}

1. Angelini P. Normal and anomalous coronary arteries: definitions and classification. Am Heart J 1989; 117: 418-34.

2. Kragel AH, Roberts WC. Anomalous origin of either the right or left main coronary artery from the aorta with subsequent coursing between aorta and pulmonary trunk: analysis of 32 necropsy cases. Am J Cardiol 1988; 62: 771-7.

3. Click RL, Holmes DR Jr, Vlietstra RE, Kosinski AS, Kronmal RA. Anomalous coronary arteries: location, degree of atherosclerosis and effect on survival-a report from the Coronary Artery Surgery Study. J Am Coll Cardiol 1989; 13: 531-7.

4. Barriales Villa R, Morís C, López Muñiz A, Hernández LC, San Román L, Barriales Alvarez V, et al. Adult congenital anomalies of the coronary arteries described over 31 years of angiographic studies in the Asturias Principality: main angiographic and clinical characteristics. Rev Esp Cardiol 2001; 54: 269-81.

5. Von Ziegler F, Pilla M, McMullan L, Panse P, Leber AW, Wilke $\mathrm{N}$, et al. Visualization of anomalous origin and course of coronary arteries in 748 consecutive symptomatic patients by 64-slice computed tomography angiography. BMC Cardiovasc Disord 2009; 9: 54.

6. Maron BJ, Doerer JJ, Haas TS, Tierney DM, Mueller FO. Sudden deaths in young competitive athletes: analysis of 1866 deaths in the United States, 1980-2006. Circulation. 2009; 119: 1085-92.

7. Maron BJ, Shirani J, Poliac LC, Mathenge R, Roberts WC, Mueller FO. Sudden death in young competitive athletes. Clinical, demographic, and pathological profiles. JAMA 1996; 276: 199-204.

8. Frescura C, Basso C, Thiene G, Corrado D, Pennelli T, Angelini A, et al. Anomalous origin of coronary arteries and risk of sudden death: a study based on an autopsy population of congenital heart disease. Hum Pathol 1998; 29: 689-95.

9. Ugalde H, Ramírez A, Ugalde D, Farías E, Silva A. [Coronary artery origin anomalies. Analysis of 10.000 coronary angiographies]. Rev Med Chile 2010; 138: 7-14.

10. Eid AH, Itani Z, Al-Tannir M, Sayegh S, Samaha A. Primary congenital anomalies of the coronary arteries and relation to atherosclerosis: an angiographic study in Lebanon. J Cardiothorac Surg 2009; 4: 58.

11. Garg N, Tewari S, Kapoor A, Gupta DK, Sinha N. Primary congenital anomalies of the coronary arteries: a coronary: arteriographic study. Int J Cardiol 2000; 74: 39-46.

12. Manning W, Li W, Cohen S, Jonson R. Improved definition of anomalous left coronary artery by magnetic resonance coronary angiography. Am Heart J 1995; 30: 615-7.

13. Lee JJ, Kim DH, Byun SS, Choi WG, Lee CW, Yi SM, et al. A case of acute myocardial infarction with the anomalous origin of the right coronary artery from the ascending 
aorta above the left sinus of Valsalva and left coronary artery from the posterior sinus of Valsalva. Yonsei Med J 2009; 50: 164-8.

14. Alessandri N, Di Matteo A, Petrassi M, Tufano F, Lanzi L, Fusco L, et al. Myocardial ischemia in cocaine-user with anomalous origin of the left circumflex coronary artery. Eur Rev Med Pharmacol Sci 2010; 14: 613-8.

15. Montaudon M, Latrabe V, Iriart X, Caix P, Laurent F. Congenital coronary arteries anomalies: review of the literature and multidetector computed tomography (MDCT) -appearance. Surg Radiol Anat 2007; 29: 343 55.

16. Davies JE, Burkhart HM, Dearani JA, Suri RM, Phillips $\mathrm{SD}$, Warnes CA, et al. Surgical management of anomalous aortic origin of a coronary artery. Ann Thorac Surg. 2009; 88: 844-7; discussion 847-8.

17. Ceyhan C, Tekten T, Onbasili AO. Primary percutaneous coronary intervention of anomalous origin of right coronary artery above the left sinus of Valsalva in a case with acute myocardial infarction. Coronary anomalies and myocardial infarction. Int J Cardiovasc Imaging 2004; 20: 293-7.

18. Azzarelli S, Amico F, Giacoppo M, Argentino V, Di Mario C, Fiscella A. Primary coronary angioplasty in a patient with anomalous origin of the right coronary artery from the left sinus of Valsalva. J Cardiovasc Med (Hagerstown) 2007; 8: 943-5.

19. Conde-Vela C, Sabaté M, Quevedo PJ, Hernández-Antolín R. Primary percutaneous coronary intervention of an anomalous right coronary artery originating from the left sinus of valsalva. Acute Card Care 2006; 8: 229-32.

20. Ramos A, Brizida L, Carvalho R, Matias F, Mourão L. Acute myocardial infarction associated with anomalous origin of coronary arteries (a rare case of origin of the left coronary artery from the ostium of the right coronary artery). Rev Port Cardiol 2010; 29: 1245-51. 